\title{
Ground State Phase Diagrams of the Extended Hubbard Model with Repulsive Pair-Hopping Interaction for Nonfrustrated 2D Lattice
}

\author{
W.R. CzART* And S. RoBAszKIEWICZ \\ Electron States of Solids Division, Faculty of Physics, Adam Mickiewicz University, \\ Umultowska 85, 61-614 Poznań, Poland
}

\begin{abstract}
We analyze the extended Hubbard model with pair-hopping interaction $J$, i.e. the Penson-Kolb-Hubbard model, in the case of repulsive $J(J<0)$ within the (broken symmetry) Hartree-Fock approximation. We focus our study on the effects of on-site $U$ interaction on the mutual stability of magnetic, charge-ordered, and superconducting states including the eta-pairing phase, i.e. the state with the Cooper-pair center-of-mass momentum $\boldsymbol{q}=\boldsymbol{Q}$. The ground state phase diagrams are obtained for arbitrary particle concentration $n$ on nonfrustrated $d=2$ square lattice.
\end{abstract}

DOI: 10.12693 /APhysPolA.127.275

PACS: 74.20.-z, 71.28. $+\mathrm{d}, 74.20 . \mathrm{Mn}$

\section{Introduction}

The Penson-Kolb-Hubbard (PKH) model is one of the conceptually simplest models for studying superconductivity with short coherence length and for description of various superconducting, magnetic and other electron orderings in narrow band systems [1-13]. The model Hamiltonian has the form

$$
\begin{aligned}
H & =-\sum_{i j \sigma} t_{i j}\left(c_{i \sigma}^{+} c_{j \sigma}+\text { H.c. }\right)-\sum_{i \sigma} \mu c_{i \sigma}^{+} c_{i \sigma} \\
& +U \sum_{i} n_{i \uparrow} n_{i \downarrow}-\frac{1}{2} J \sum_{\langle i j\rangle}\left(c_{i \uparrow}^{+} c_{i \downarrow}^{+} c_{j \downarrow} c_{j \uparrow}+\text { H.c. }\right),
\end{aligned}
$$

where $n_{i \sigma}=c_{i \sigma}^{+} c_{i \sigma}, t_{i j}$ is the single electron hopping integral, $U$ is the on-site density-density interaction, $J$ is the pair hopping (intersite charge exchange) interaction, $\mu$ is the chemical potential. The limit $\langle i j\rangle$ restricts the sum to nearest neighbors (nn) and $n=\frac{1}{N} \sum_{i \sigma}\left\langle n_{i \sigma}\right\rangle$.

The model includes a nonlocal pairing mechanism (the pair hopping term $J$ ) that is distinct from the on-site interaction in the attractive Hubbard $(\mathrm{AH})$ model [1416] and that is the driving force of pair formation and also of their condensation.

In the following we will focus on the case of repulsive $J$ $(J<0)$ which favorize superconducting eta-pairing order in the system. The case of attractive $J(J>0)$ will be discussed in a separate paper.

As in Ref. [2] our analysis is based on the (broken symmetry) HFA. In the derivation of the eigensolutions we have assumed an alternated lattice, i.e. $\epsilon_{k+Q}=-\epsilon_{k}$. For arbitrary electron concentration $n$ the stable solutions are determined as the minimum of the free energy of the system $F$ with respect to the variational parame-

* corresponding author; e-mail: czart@amu.edu.pl ters $x_{\alpha}(\alpha=\mathrm{AF}, \mathrm{F}, \mathrm{CO}, \mathrm{S}$, eta $)$ and $\mu$, i.e. by the equations

$$
\frac{\partial F}{\partial x_{\alpha}}=0 \text { and } \frac{\partial F}{\partial \mu}=0,
$$

from which we get sets of self-consistent equations for each considered ordering type. The order parameters for considered phases:

(i) antiferromagnetic (AF):

$$
x_{\mathrm{AF}}=\frac{1}{2 N} \sum_{i, \sigma} \sigma \exp \left(\boldsymbol{Q}_{i} \cdot \boldsymbol{R}_{i}\right)\left\langle c_{i \sigma}^{+} c_{\iota \sigma}\right\rangle,
$$

(ii) ferromagnetic $(\mathrm{F})$ :

$$
x_{\mathrm{F}}=\frac{1}{N} \sum_{i, \sigma} \sigma\left\langle c_{i \sigma}^{+} c_{\iota \sigma}\right\rangle,
$$

(iii) charge ordered $(\mathrm{CO})$ : $x_{\mathrm{CO}}=\frac{1}{2 N} \sum_{i, \sigma} \exp \left(\boldsymbol{Q}_{i} \cdot \boldsymbol{R}_{i}\right)\left\langle c_{i \sigma}^{+} c_{\iota \sigma}\right\rangle$,

(iv) s-wave pairing superconductivity (S): $x_{s}=\frac{1}{N} \sum_{i}\left\langle c_{i \downarrow} c_{\iota \uparrow}\right\rangle=\frac{1}{N} \sum_{k}\left\langle c_{-k \downarrow} c_{k \uparrow}\right\rangle$,

(v) eta-pairing superconductivity (eta): $x_{\eta}=\frac{1}{N} \sum_{i} \exp \left(\boldsymbol{Q}_{i} \cdot \boldsymbol{R}_{i}\right)\left\langle c_{i \downarrow} c_{\iota \uparrow}\right\rangle$, where $\boldsymbol{Q}=\frac{\Pi}{a}, \frac{\Pi}{a}, \ldots$

\section{Results and comments}

We have performed analysis of the ground state phase diagrams of the model (1) for square (SQ) lattice and arbitrary electron concentration $n(0<n<2)$ involving magnetic orderings, for repulsive $U(U>0)$, charge orderings, for attractive $U(U<0)$ and the superconducting states for $U>0$ and $U<0$, in the case of repulsive $J(J<0)$. Due to the electron-hole symmetry of the system all the plotted diagrams are symmetric under the transformation $n \rightarrow 2-n$.

In Figs. 1, 2 the phase diagrams were plotted for the case of repulsive $U$, whereas Figs. 3 and 4 are for attractive $U$. In all presented diagrams the transitions to nonordered phase $(\mathrm{N})$ are of the second order, and the transitions between ordered states are of the first order. Dashed lines in the figures determine the borders between strong- and weak-eta phases commented in the final part of our report. 


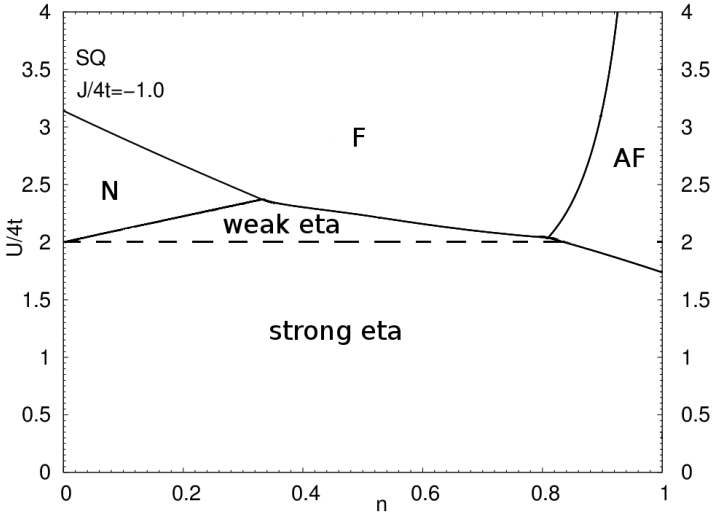

Fig. 1. The ground state phase diagram as a function of repulsive on-site interaction $U$ and carriers concentration $n$ for fixed $J / 4 t=-1$. Denotations of phases: nonordered $(\mathrm{N})$, ferromagnetism $(\mathrm{F})$, antiferromagnetism (AF) and eta-pairing superconducting state (eta). All diagrams are plotted for nonflustrated $2 \mathrm{D}$ square lattice (SQ).

In Fig. 1 we present the phase diagram of the system for fixed $J / 4 t=-1$ as a function of repulsive interaction $U(U>0)$ and concentration $n$.

In the diagram as on-site interaction $U$ is increased one can observe several different types of behavior, depending on carriers concentration $n$ : (i) a sequence of second-order transitions eta-N-F, (ii) a first-order transition eta-F, (iii) a sequence of first-order transitions etaAF-F and (iv) at half-filling a single first-order transition eta-AF. As we can see in Fig. 1 the eta-phase is stable below a certain critical value $U_{\mathrm{c}}$ which depends on concentration $n$. The value of $U_{\mathrm{c}}$ increases with increasing $|J|$. As it is illustrated on the plot, superconducting eta phase can occur in the whole range of concentration $n(0<n<2)$ only below certain characteristic value of on-site interaction $U<U_{\mathrm{c} 1}$ dependent on $J\left(U_{\mathrm{c} 1}<U_{\mathrm{c}}\right)$. With increasing $|J|$, the value $U_{\mathrm{c} 1}$ increases.

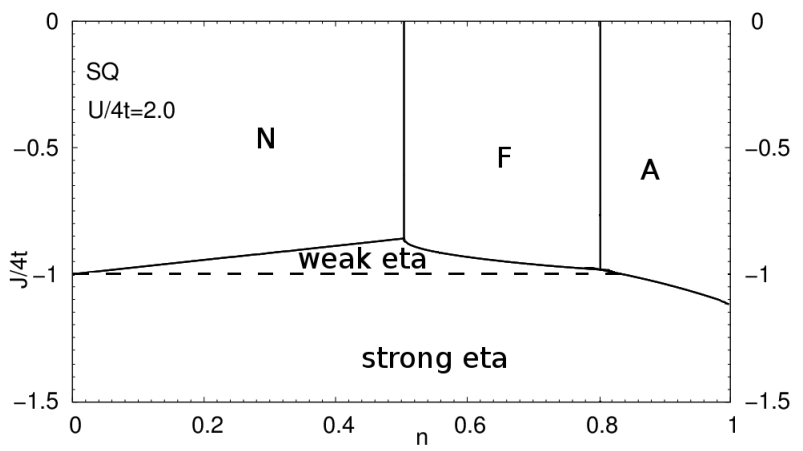

Fig. 2. The ground state phase diagram as a function of $J$ and $n$ for fixed $U / 4 t=2$. Denotations as in Fig. 1.

An example of the ground state $J$ vs. $n$ phase diagram for fixed $U>0(U / 4 t=2)$ is presented in Fig. 2 .
In general the eta-phase is stable above a certain critical value $\left|J_{\mathrm{c}}\right|$ which depends on concentration $n$ and which increases with increasing $U$. For large $|J|$ superconducting eta-phase is stable within the whole range of concentration $n(0<n<2)$. For smaller $|J|$ the following transitions can occur with increasing $n$ : (i) a first-order transition eta-AF, (ii) a sequence of second-order transition $\mathrm{N}$-eta and a first-order transition eta-AF, (iii) a sequence of second-order transition N-eta and two first-order transitions eta-F-AF, (iv) a sequence of second-order transition N-F and a first-order transitions F-AF and finally (v) a first-order transitions F-AF.

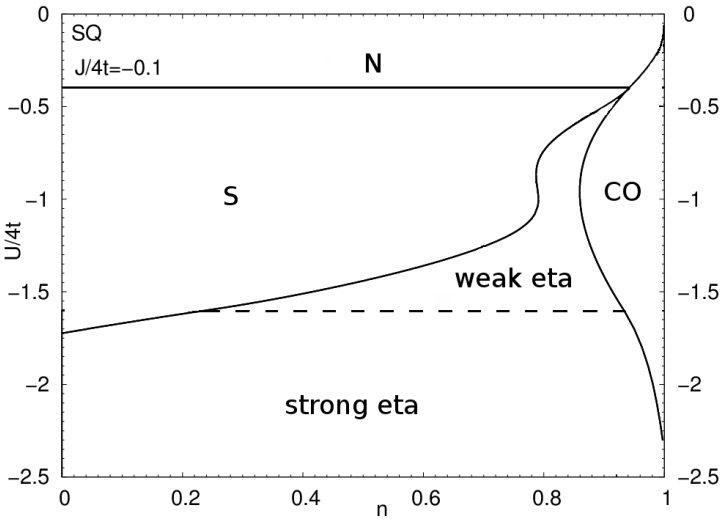

Fig. 3. The ground state phase diagram as a function of attractive on-site interaction $U$ and concentration $n$ for fixed $J / 4 t=-0.1$. Denotations of phases: chargeordered (CO), s-wave superconducting (S), eta-pairing superconducting (eta) and nonordered (N).

In Fig. 3 we show the ground state phase diagram of the system as a function of attractive $U(U<0)$ and $n$ for fixed $J / 4 t=-0.1$, whereas Fig. 4 is an example of the $J$ vs. $n$ diagram for fixed $U<0(U / 4 t=-1)$. In the system with attractive interaction $U$ the charge-ordered phase and $s$-wave pairing superconductivity can be stable in definite ranges of $n$ and $U$. With decreasing $|U|$ (Fig. 3), we observe the following series of transitions for given $n$ : (i) a sequence of first-order transition eta-S and second-order S-N, (ii) a reentrant eta and S phenomenon in a sequence of first-order transitions eta-S-eta-S and second-order transition S-N, (iii) a reentrant eta phenomenon in a sequence of first-order transitions eta-COeta-S and second-order transition $\mathrm{S}-\mathrm{N}$, (iv) a reentrant eta phenomenon in a sequence of first-order transition eta-CO and second-order transition eta-N, (v) a sequence of first-order transition eta- $\mathrm{CO}$ and second-order transition $\mathrm{CO}-\mathrm{N}$ and (vi) at the half-filling: a single first-order transition between superconducting and charge ordered states (eta-CO).

Due to competition between attractive $U$ and repulsive $J$ the S phase can be stable in the system only below a critical value of $|U|<U_{c}$ which depends on concentration $n$ and interaction $J$ (cf. Figs. 3, 4). In general $\mathrm{CO}$ and $\mathrm{S}$ phases occur within a limited range of $|U|$ 
and $|J|$ and CO phase can be realized only close to $n=1$ and at half filling. Two reentrant eta phenomena seen in the diagram of Fig. 3, result from the competition between (i) s-wave and eta-pairing superconducting states and (ii) eta-pairing superconducting and charge-ordered states.

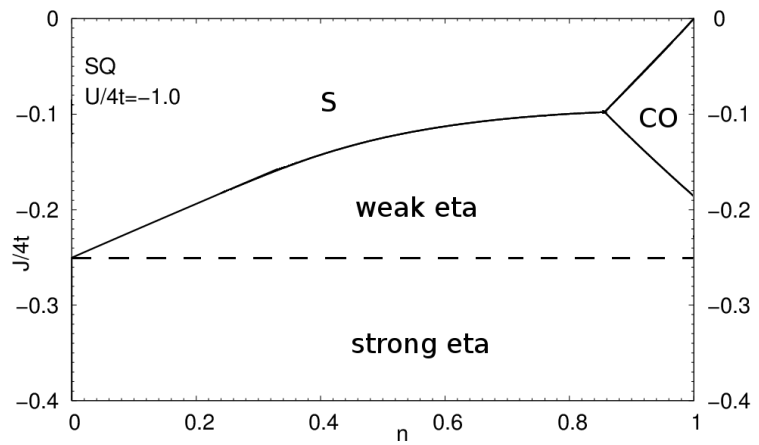

Fig. 4. The ground state phase diagram as a function of $J$ and $n$ for fixed $U / 4 t=-1$. Denotations as in Fig. 3 .

As we have found, in the model considered the eta pairing state can be stable only above some critical values of repulsive $|J|$ depending on $U$ and $n$. The system in this state never exhibits standard BCS features [2, 17, 18]. This behaviour is in contrast, with the properties of the isotropic $s$-wave state, which for $U \leq 0$ is stable for any attractive $J<0$, and the system exhibits a smooth crossover from the BCS-like limit to the tightly bound pairs regime with increasing $J[2,7,14-16,19]$.

The electronic spectrum of the eta phase consists of two branches $E_{k}^{+}$and $E_{k}^{-}$and the minimum gap between the lower and higher band $E_{g}^{\min }=\min E_{k}^{+}-\max E_{k}^{-}$can be either positive or negative, depending on interaction parameters and temperature. For $E_{\mathrm{g}}^{\min }>0$ the order parameter of the eta phase at $T=0$ takes its maximum value (the same as in the zero bandwidth limit) $x_{\eta}^{\max }=\frac{1}{2} \sqrt{n(2-n)}[8,17,18]$. We define this state as the strong eta-pairing phase (in analogy with strong ferromagnet). For $E_{\mathrm{g}}^{\min } \leq 0$ and $x_{\eta}<x_{\eta}^{\max }$ at $T=0$ we define this state as the weak eta-pairing state (in analogy with weak ferromagnet). The borders between strong and weak-eta pairing states are drawn in Figs. 1-4 as dashed lines. As we see the weak-eta phase exists only in a restricted range of $n$ which shrinks to zero with decreasing $|J|$ (cf. Fig. 2 and Fig. 4) and with increasing $U$ (cf. Fig. 1 and Fig. 3).

The results concerning the finite temperature properties of the model [18] show that the energy gaps $E_{\mathrm{g}}^{\min }$ existing at $T=0$ in the strong-eta phase are reduced with increasing $T$ and vanish at some characteristic temperature $T_{m}$ being always lower (except $t=0$ ) than $T_{\mathrm{c}}$ determining transition to the nonordered state. Thus, even for the strong-eta phase there exists a range of $T$ where the system with eta pairing will exhibit a gapless behavior.
In this report we have concentrated on the ground state phase diagrams for the $\mathrm{PKH}$ model with repulsive pair hopping interaction. In our analysis we have taken into account only simple types of various homogeneous electron orderings. Extended work with the analysis of thermodynamic properties of the model and considering mixed orderings and phase separated states is in preparation [18].

\section{Acknowledgments}

We thank P. Grzybowski, K. Kapcia and R. Micnas for helpful discussions.

\section{References}

[1] A. Hui, S. Doniach, Phys. Rev. B 48, 2063 (1993).

[2] S. Robaszkiewicz, B. Bułka, Phys. Rev. B 59, 6430 (1999) and references therein; A. Ptok, D. Crivelli, K.J. Kapcia, Supercond. Sci. Technol. 28, 045010 (2015).

[3] G.I. Japaridze, E. Muller-Hartmann, J. Phys. Condens. Matter 9, 10509 (1997); G.I. Japaridze, A.P. Kampf, M. Sekania, P. Kakashvili, Ph. Brune, Phys. Rev. B 65, 014518 (2001).

[4] K.A. Penson, M. Kolb, Phys. Rev. B 33, 1663 (1986).

[5] R. Micnas, J. Ranninger, S. Robaszkiewicz, Rev. Mod. Phys. 62, 113 (1990).

[6] G.K. Roy, B. Bhattacharyya, Phys. Rev. B 55, 15506 (1997).

[7] S. Robaszkiewicz, W. Czart, Acta Phys. Pol. B 32, 3267 (2001); W.R. Czart, S. Robaszkiewicz, Acta Phys. Pol. A 97, 217 (2000); W.R. Czart, S. Robaszkiewicz, Acta Phys. Pol. A 100, 885 (2001).

[8] W.R. Czart, S. Robaszkiewicz, Acta. Phys. Pol. A 106, 709 (2004); W.R. Czart, S. Robaszkiewicz, Acta Phys. Pol. A 127, 278 (2015).

[9] M. Mierzejewski, M. Maśka, Phys. Rev. B 69, 054502 (2004); A. Ptok, M. Maśka , M. Mierzejewski, J. Phys. Condens. Matter 21, 045703 (2009).

[10] K. Kapcia, S. Robaszkiewicz, R. Micnas, J. Phys. Condens. Matter 24, 215601 (2012).

[11] K. Kapcia, J. Supercond. Nov. Magn. 27, 913 (2014).

[12] K. Kapcia, S. Robaszkiewicz, J. Phys. Condens. Matter 25, 065603 (2013); K.J. Kapcia, Acta Phys. Pol. A 126, A-53 (2014).

[13] K. Kapcia, Acta Phys. Pol. A 121, 733 (2012); K. Kapcia, J. Supercond. Nov. Magn. 26, 2647 (2013).

[14] W. Czart, T. Kostyrko, S. Robaszkiewicz, J. Magn. Magn. Mater. 140-144, 2059 (1995).

[15] W. Czart, T. Kostyrko, S. Robaszkiewicz, Physica C 272, 51 (1996).

[16] B. Bułka, S. Robaszkiewicz, Phys. Rev. B 54, 13138 (1996).

[17] S. Robaszkiewicz, W. Czart, Phys. Status Solidi B 236, 416 (2003); K.J. Kapcia, J. Supercond. Novel Magn. in press (2015); DOI:10.1007/s10948014-2906-4

[18] W.R. Czart, S. Robaszkiewicz, in preparation.

[19] W. Czart, S. Robaszkiewicz, Phys. Rev. B 64, 104511 (2001). 